\title{
Management of Arteriovenous malformation arising from Hemangiopericytoma
}

\section{S Vinod Thangaswamy ${ }^{1}$, Prasanna Paul ${ }^{2}$, Shreya B Malapur ${ }^{3}$}

1. Dr. S Vinod Thangaswamy, Professor, Department of oral and maxillofacial surgery, Coorg Institute of Dental Sciences, Virajpet

2. Dr. Prasanna Paul, Consultant, Oral and Maxillofacial Surgeon, Banglore

3. Dr. Shreya B Malapur, Post-graduate, Department of oral and maxillofacial surgery, Coorg Institute of Dental Sciences, Virajpet

Corresponding author:

Dr. S Vinod Thangaswamy

Professor

Department of oral and maxillofacial surgery

Coorg Institute of Dental Sciences, Virajpet

Phone: 9791642614

\begin{abstract}
Identification and treatment of vascular malformations is a challenging endeavor for clinician. Hemangiopericytoma is highly malignant over a life time, the recurrence rate varies with the particular organsystem involved. Arteriovenous malformations (AVMs) are high-flow malformation, characterized by direct communication between arteries and veins, lacking normal capillary network. AVMs are usually congenital. Acquired AVMs are reported to occur due to trauma or hormonal changes.

The goal of this article is to provide a management of arteriovenous malformations arising from hemangiopericytoma. Only by developing a clear understanding of the clinical aspects, diagnostic tools, imaging modalities, and options for surgical intervention should be taken with appropriate care be provided.
\end{abstract}

Key words: Arteriovenous malformation, Hemangiopericytoma, Extraoral Swelling 


\section{Introduction}

Vascular malformations are asymptomatic lesions, found within the bony skeleton; they do not regress with age and may be associated with severe life-threatening hemorrhage ${ }^{1}$.

Hemangiopericytoma is a rare vascular tumor composed of capillaries bounded by peculiar, elongated, and contractile cells, first described by Zimmerman as pericytes. Hemangiopericytomas can be either begin or malignant, seen in all age groups with equal gender distribution, and have a high chance for metastasis ${ }^{2}$. The clinical series have confirmed the widespread distribution of these tumors ${ }^{3,4}$.

Arteriovenous malformation (AVM) is a congenital vascular malformation with direct communications from arteries to veins with lack of normal capillary network5. The peripheral or extra cranial AVM are usually locally aggressive causing cosmetic and functional disturbance. AVM if untreated can destroy normal tissues and can lead to complications such as severe disfigurement, uncontrollable bleeding, ulceration, pain and cardiac volume overload6.

We present a case report of hemangiopericytoma with incomplete primary surgical excision had resulted in secondary AV malformation arising out of the lesion .

\section{Case Report}

A 42-year-old male patient reported with chief complaint of a swelling on the upper right side of the face above the cheek and lateral to right eye since 2 years. Patient had history of swelling since childhood and had undergone surgery for the same 2 years ago. Following the surgery there was a recurrence of the swelling, which was gradual in onset, progressively increased in size, not associated with pain and systemic condition.

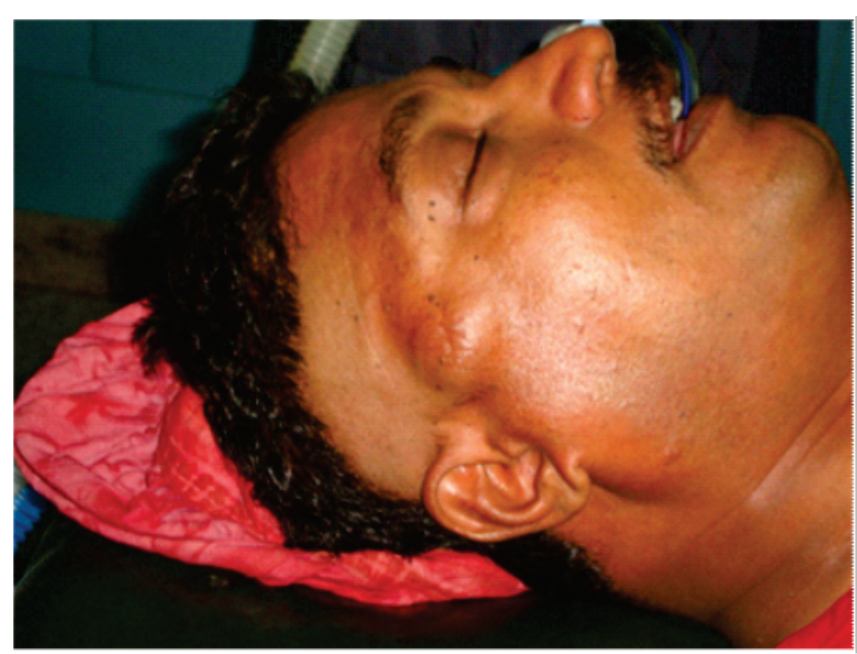

FIG. 1 - SHOWING DIFFUSE SWELLING ON RIGHT TEMPORAL REGION OF FACE APPROXIMATELY 3.5X3 CM WITH PRESENCE OF CENTRAL SURGICAL SCAR

On examination, swelling was diffuse with a size of approximately $3.5 \times 3 \mathrm{~cm}$, extending superioinferiorly from upper border of orbital region to ala of the nose. Anterioposteriorly from lateral orbital region to anterior of the tragus, surgical scar was present. On palpation swelling was pulsatile in nature, non-tender and no increase in local rise in temperature noted and all inspectory findings were confirmed.

Based on the history and clinical findings, a provisional diagnosis of $\mathrm{AV}$ malformation (VM) was made, and in differential diagnosis, pyogenic granuloma and vascular tumors were considered.

Complete hemogram revealed all the values within normal limits, Electrocardiogram was normal, CT angiography was done to check for the feeder arteries, which showed the lesion was of low flow type with predominant feeders from superficial temporal artery.

The treatment plan was surgical excision of the lesion. Intraoperatively ligation of superficial temporal artery and its collateral feeders to the lesion were performed, followed by surgical excision of the lesion in total. Reconstruction of the surgical defect was performed by using temporalis fascia and muscle coverage which was done under general anesthesia. 


\section{$J i n$ ?}

J. Multi Dent Res. 2019: 5(1); 42-46

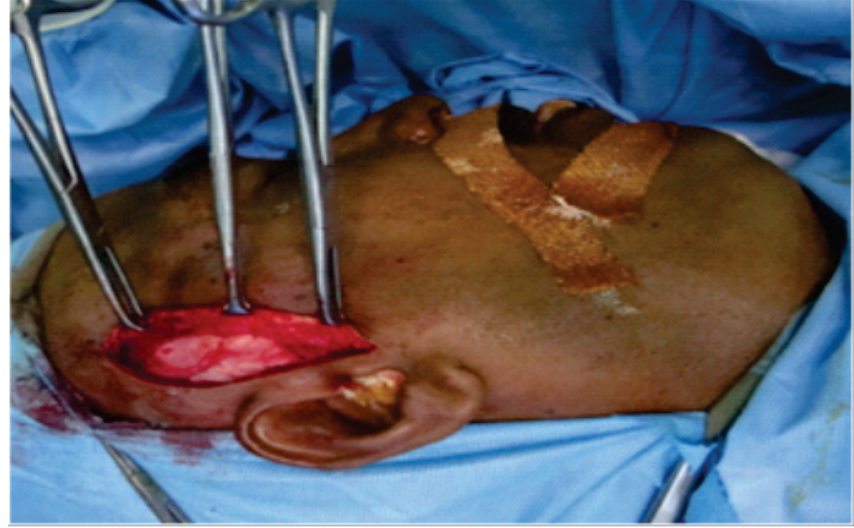

FIG. 2 - ALKAYAT BRAMELY INCISION GIVEN FOR SURGICAL EXPOSURE OF LESION

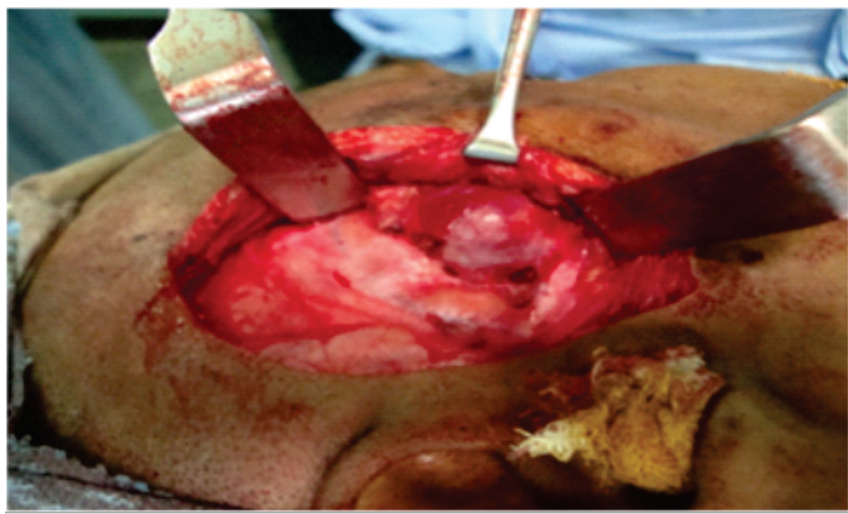

FIG. 3 - EXPOSURE OF THE LESION

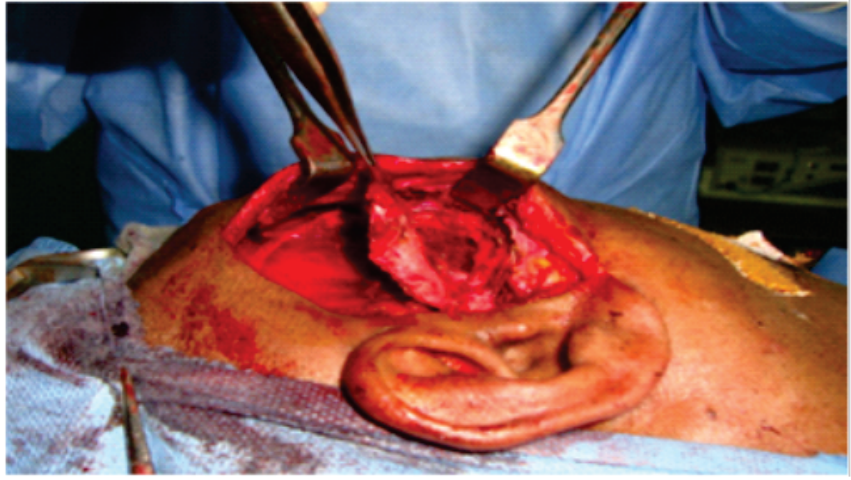

FIG. 4 - DEAD SPACE SEEN AFTER SURGICAL EXCISION OF LESION

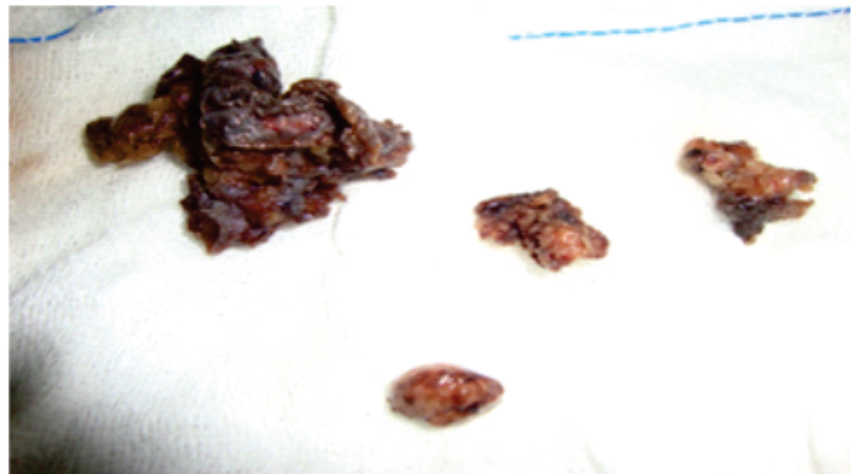

FIG. 5 - COMPLETE EXCISION OF THE LESION

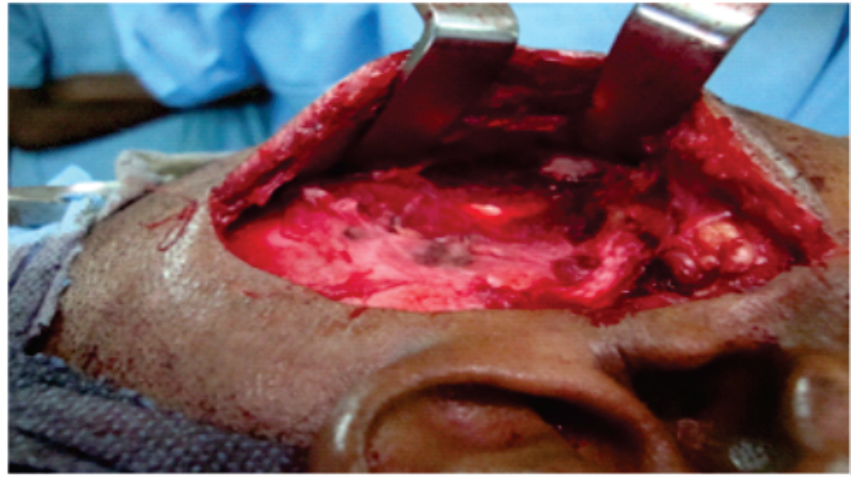

FIG. 6 - RECONSTRUCTION WITH TEMPORALIS FASCIA AND MUSCLE

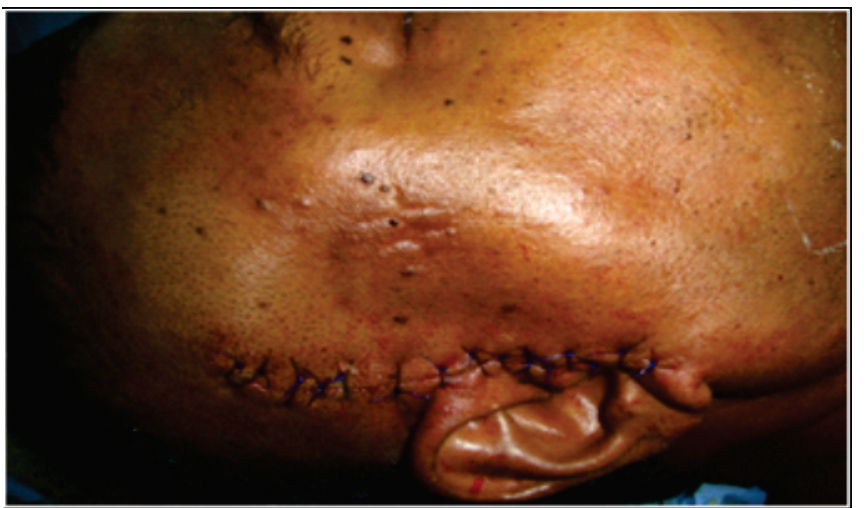

FIG. 7- CLOSURE OF THE SURGICAL SITE

The patient when reviewed after a month, surgical wound had healed completely. The patient was asymptomatic when reviewed after a year.

\section{Discussion}

This case report presents a extracranial hemangiopericytoma associated with AVMs. Cases of hemangiopericytomas in association of AVMs have been reported6. To our knowledge, however, a hemangiopericytoma of this intensity in association with AVMs in head and neck has not been reported.

According to biological classification of congenital vascular malformations given by Mulliken and Glowacki it is based on the main pathological characteristics of the endothelium and the natural course of the lesion. Later this classification was redefined by Mulliken and Young and taken by the International Society for the Study of Vascular Anomalies (ISSVA) in 1996; today it is 
the most widely accepted classification with minimal changes to the original version which states 7. Vascular malformations are localised defects of vascular morphogenesis and should be differentiated from hemangiomas, which are true neoplasms of endothelial cells ${ }^{8}$.

Lesions of vascular tissue in head and neck are of importance to Oral and Maxillofacial surgeons. Vascular lesions, which are broadly divided into Hemangiomas and Vascular malformations, can result in significant cosmetic problems for the patient. AV malformation is a vascular lesion that exhibits high flow resulting in severe hemorrhage with significant morbidity'.

Hemangiopericytoma is a rare vascular tumor composed of capillaries bounded by peculiar, elongated, and contractile cells, 'first described by zimmerman as pericytes Total incidence of VM is about 1 in 10000. These lesions usually occur in the head and neck with a predilection in the oral cavity, muscle groups and airway ${ }^{10}$.

In this case, patient had undergone surgery for Hemagiopericytoma, for which incomplete primary surgical excision had resulted in the formation of secondary AV malformation arising out from remnant lesion. The treatment of surgical excision was performed where complete removal of lesion with reconstruction of temporalis fascia and muscle was followed.

Careful meticulous pre-surgical workup in the diagnosis of the lesion with the recent available diagnostic aids (CT angiography with contrast) for identification of the feeder vessels will help in definitive and complete surgical addressal of the lesion.

\section{Conclusion}

Acquired AVMs are very rare in the head and neck region. They present cosmetic and functional disturbances. They may result in alarming hemodynamic manifestations such as venous engorgement, distal ischemia and high-output cardiac failure. Hence, early diagnosis and treatment of such cases is must. A good clinical history with imaging usually delineates the lesion well and gives a chance to make a careful decision of intervention. The surgical management is complex which requires elaborate planning and multidisciplinary approach. This case report provides surgical management of secondary AVMs arising from hemangiopericytoma in the head and neck.

\section{References}

1. Baglio, C. M., and Crowson, C. N.: Hemangiopericytoma of urachus; report of a case. J. Urol. 91:66\&662, 1964.

2. B a ckwinkel K D, Diddam s J A : Hemangiopericytoma: Report of a case and comprehensive review of the literature. Cancer 25:896-901, 1970.

3. Large Hemangiopericytoma Associated with Arteriovenous Malformations and Dural Arteriovenous Fistulae. World Neurosurg. (2011) 76, 6:592.e7-592.e10. DOI: 10.1016/j.wneu.2011.05.023

4. Hemangiopericytoma of the Head and NeckKathleen R. Billings, MD,* Yao S. Fu, $\mathrm{MD}, \uparrow$ Thomas C. Calcaterra, MD, ${ }^{*}$ and Joel A. Sercarz, MD*+.J Otolaryngol 2000;21:238243.

5. Vascular Malformations That Were Diagnosed as or Accompanied by Malignant Tumors Seiji Houbara, MD, Sadanori Akita, MD, PhD, Hiroshi Yoshimoto, MD, $\mathrm{PhD}$, and Akiyoshi Hirano, MD*.Dermatol Surg 2014 ; 40:1225-1232.

6. Ecker RD, Marsh WR, Pollock BE, KurtkayaYapicier O, McClelland R, Scheithauer BW, 
Buckner JC: Hemangiopericytoma in the central nervous system: treatment, pathological features, and longterm follow up in 38 patients. $\mathrm{J}$ Neurosurg 98:1182-1187, 2003.

7. Espat NJ, Lewis JJ, Leung D, Woodruff JM, Antonescu CR, Shia J, Brennan MF: Conventional hemangiopericytoma: modern analysis of outcome. Cancer 95:1746-1751, 2002.

8. McMaster MJ, Soule EH, Ivins JC: Hemangiopericytoma. A clinic0- pathologic study and long-term follow up of 60 patients. Cancer 36:2232-2244, 1975.

9. McMaster MJ, Soule EH, Ivins JC: Hemangiopericytoma: A clinicopathologic study and long-term followup of 60 patients. Cancer 36:2232-2244, 1975

10. Acquired arteriovenous malformation of lip occurring as an occupational hazard: A case report with review of literature $\mathrm{J}$ omfp 22;2$287,2018$. 